\title{
A Tableau Proof System with Names for Modal Mu-calculus
}

\author{
Colin Stirling ${ }^{1}$ \\ School of Informatics \\ University of Edinburgh \\ Edinburgh, UK \\ cps@inf.ed.ac.uk
}

\begin{abstract}
Howard Barringer was a pioneer in the study of temporal logics with fixpoints 11. Their addition adds considerable expressive power. One general issue is how to define proof systems for such logics. Here we examine proof systems for modal logic with fixpoints. We present a tableau proof system for checking validity of formulas which uses names to keep track of unfoldings of fixpoint variables as devised in [8].
\end{abstract}

\section{Introduction}

Howard Barringer was a pioneer in the study of temporal logics with fixpoints. Their addition adds considerable expressive power. In [1] Banieqbal and he present a semantic tableau decision procedure for checking satisfiability of formulas of linear time temporal logic with fixpoints. They show that a formula is satisfiable if, and only if, it is satisfiable in an eventually cyclic model. They then employ a marking algorithm on graphs whose nodes are sets of satisfiable formulas to ensure that a least fixpoint formula is only unfolded finitely often.

In this paper we consider modal mu-calculus, modal logic with fipoints, see [2] for a survey. Here our interest is more with developing proof systems for the logic. In this paper we describe a tableau proof system which checks when a modal mu-calculus formula is valid. The system uses names to keep track of unfoldings of fixpoint variables. This idea originated in 10 in the context of model checking. For satisfiability checking it was used in [7] for LTL and CTL and then for modal mu-calculus in 8 .

In Section 2 we describe the syntax and semantics of modal mu-calculus and in Section 3 we briefly examine approaches to devising proof systems for this logic. The tableau proof system based on names for checking valid formulas is then presented in Section 4 and shown to be both sound and complete.

\section{Modal Mu-calculus}

Let Var be an (infinite) set of variable names, typically indicated by $Z, Y, \ldots$; let Prop be a set of atomic propositions, typically indicated by $P, Q, \ldots$; and let Act be a set of actions, typically indicated by $a, b, \ldots$. The set of modal mu-calculus formulas $\mu M$ (with respect to Var, Prop, Act) is as follows.

$$
\phi::=Z|P| \neg \phi\left|\phi_{1} \wedge \phi_{2}\right|[a] \phi \mid \nu Z . \phi
$$

In $\nu Z . \phi$ every free occurrence of $Z$ in $\phi$ occurs positively, that is within the scope of an even number of negations. If a formula is written as $\phi(Z)$, it is to be understood that the subsequent writing of $\phi(\psi)$ means $\phi$ with $\psi$ substituted for all free occurrences of $Z$. 
The positivity requirement on the fixpoint operator is a syntactic means of ensuring that $\phi(Z)$ denotes a functional monotonic in $Z$, and so has unique minimal and maximal fixed points. It is usually more convenient to introduce derived dual operators, and work in positive form: $\phi_{1} \vee \phi_{2}$ means $\neg\left(\neg \phi_{1} \wedge \neg \phi_{2}\right),\langle a\rangle \phi$ means $\neg[a] \neg \phi$ and $\mu Z$. $\phi(Z)$ means $\neg \nu Z . \neg \phi(\neg Z)$. A formula is in positive form if it is written with the derived operators so that $\neg$ only occurs applied to atomic propositions. It is in positive normal form if in addition all bound variables are distinct. Any closed formula can be put into positive normal form. It is also useful to have derived propositional constants tt (for $P \vee \neg P$ ) and ff (for $P \wedge \neg P$ ).

A modal mu-calculus structure $\mathrm{T}$ (over Prop, Act) is a labelled transition system, namely a set $\mathrm{S}$ of states and a family of transition relations $\stackrel{a}{\longrightarrow} \subseteq \mathrm{S} \times \mathrm{S}$ for $a \in$ Act, together with an interpretation $\bigvee_{\text {Prop }}$ : Prop $\rightarrow 2^{\mathrm{S}}$ for the atomic propositions. As usual we write $s \stackrel{a}{\longrightarrow} t$ for $(s, t) \in \stackrel{a}{\longrightarrow}$.

Given a structure $T$ and an interpretation $V$ : Var $\rightarrow 2^{S}$ of the variables, the set $\|\phi\|_{V}^{\top}$ of states satisfying a formula $\phi$ is defined as follows:

$$
\begin{aligned}
\|P\|_{\mathrm{V}}^{\top} & =\mathrm{V}_{\text {Prop }}(P) \\
\|Z\|_{\mathrm{V}}^{\top} & =\mathrm{V}(Z) \\
\|\neg \phi\|_{\mathrm{V}}^{\top} & =\mathrm{S}-\|\phi\|_{\mathrm{V}}^{\top} \\
\left\|\phi_{1} \wedge \phi_{2}\right\|_{\mathrm{V}}^{\top} & =\left\|\phi_{1}\right\|_{\mathrm{V}}^{\top} \cap\left\|\phi_{2}\right\|_{\mathrm{V}}^{\top} \\
\|[a] \phi\|_{\mathrm{V}}^{\top} & =\left\{s \mid \forall t . \text { if } s \stackrel{a}{\longrightarrow} t \text { then } t \in\|\phi\|_{\mathrm{V}}^{\top}\right\} \\
\|\nu Z . \phi\|_{\mathrm{V}}^{\top} & =\bigcup\left\{S \subseteq \mathrm{S} \mid S \subseteq\|\phi\|_{\mathrm{V}[Z:=S]}^{\top}\right\}
\end{aligned}
$$

where $\mathrm{V}[Z:=S]$ is the valuation which maps $Z$ to $S$ and otherwise agrees with $\mathrm{V}$. If we are working in positive normal form, we may add definitions for the derived operators by duality (and for the propositional constants).

$$
\begin{aligned}
\left\|\phi_{1} \vee \phi_{2}\right\|_{\mathrm{V}}^{\top} & =\left\|\phi_{1}\right\|_{\mathrm{V}}^{\top} \cup\left\|\phi_{2}\right\|_{\mathrm{V}}^{\top} \\
\|\langle a\rangle \phi\|_{\mathrm{V}}^{\top} & =\left\{s \mid \exists t . s \stackrel{a}{\longrightarrow} t \wedge t \in\|\phi\|_{\mathrm{V}}^{\top}\right\} \\
\|\mu Z . \phi\|_{\mathrm{V}}^{\top} & =\bigcap\left\{S \subseteq \mathrm{S} \mid S \supseteq\|\phi\|_{\mathrm{V}[Z:=S]}^{\top}\right\} \\
\|\mathrm{tt}\|_{\mathrm{V}}^{\top} & =\mathrm{S} \\
\|\mathrm{ff}\|_{\mathrm{V}}^{\top} & =\emptyset
\end{aligned}
$$

If we take the usual lattice struture on $2^{\mathrm{S}}$, given by set inclusion, and if $f$ is a monotonic function then by the Knaster-Tarski theorem $f$ has fixed points, and indeed has a unique maximal and a unique minimal fixed point. The maximal fixed point is the union of post-fixed points, $\{S \subseteq \mathrm{S} \mid S \subseteq f(S)\}$, and the minimal fixed point is the intersection of pre-fixed points, $\bigcap\{S \subseteq \mathrm{S} \mid f(S) \subseteq S\}$. These determine the meanings of $\nu$ and $\mu$ in $\mu M$.

Moreover, the standard theory of fixpoints tells that if $f$ is a monotone function on a lattice, we can construct its minimal fixed point by applying $f$ repeatedly on the least element of the lattice to form an increasing chain, whose limit is the least fixed point. Similarly, the maximal fixed point is constructed by applying $f$ repeatedly on the largest element to form a decreasing chain, whose limit is the maximal fixed point. The stages of these iterations can be introduced syntactically as $\mu^{\alpha} Z . \phi$ and $\nu^{\alpha} Z$. $\phi$ for ordinals $\alpha$ whose meanings are as follows when $\lambda$ is a limit ordinal. 


$$
\begin{aligned}
\left\|\mu^{0} Z . \phi\right\|_{\mathrm{V}}^{\top} & =\emptyset \\
\left\|\nu^{0} Z . \phi\right\|_{\mathrm{V}}^{\top} & =\mathrm{S} \\
\left\|\mu^{\beta+1} Z . \phi\right\|_{\mathrm{V}}^{\top} & =\left\|\phi\left(\mu^{\beta} Z . \phi\right)\right\|_{\mathrm{V}}^{\top} \\
\left\|\nu^{\beta+1} Z . \phi\right\|_{\mathrm{V}}^{\top} & =\left\|\phi\left(\nu^{\beta} Z . \phi\right)\right\|_{\mathrm{V}}^{\top} \\
\left\|\mu^{\lambda} Z . \phi\right\|_{\mathrm{V}}^{\top} & =\bigcup_{\beta<\lambda}\left\|\mu^{\beta} Z . \phi\right\|_{\mathrm{V}}^{\top} \\
\left\|\nu^{\lambda} Z . \phi\right\|_{\mathrm{V}}^{\top} & =\bigcap_{\beta<\lambda}\left\|\nu^{\beta} Z . \phi\right\|_{\mathrm{V}}^{\top}
\end{aligned}
$$

Definition 1. The formula $\phi$ of $\mu M$ is valid if for all structures $\mathrm{T}$ and interpretations $\mathrm{V}$, $\|\phi\|_{\mathrm{V}}^{\mathrm{\top}}=\mathrm{S}$. The formula $\phi$ is satisfiable if there is a structure $\mathrm{T}$ and an interpretation $\mathrm{V}$ such that $\|\phi\|_{\vee}^{\top} \neq \emptyset$.

As is standard $\models \phi$ indicates that $\phi$ is valid and $s \in\|\phi\|_{\mathrm{V}}^{\mathrm{T}}$ is written as $s \models_{(\mathrm{T}, \mathrm{V})} \phi$, dropping the index $(\mathrm{T}, \mathrm{V})$ wherever possible.

The relationship between stages of iteration and the fixpoints is formally described.

Fact 1. 1. $s=\nu Z . \phi$ iff $s \models \nu^{\alpha} Z . \phi$ for all ordinals $\alpha$.

2. $s \models \mu Z$. $\phi$ iff $s \models \mu^{\alpha} Z$. $\phi$ for some ordinal $\alpha$.

So for a minimal fixpoint formula $\mu Z . \phi$, if $s$ satisfies the fixpoint, it satisfies some iterate, say the $\beta+1$ th so that $s=\mu^{\beta+1} Z$. $\phi$. Now if we unfold this formula once, we get $s=\phi\left(\mu^{\beta} Z . \phi\right)$. Therefore, the fact that $s$ satisfies the fixpoint depends, via $\phi$, on the fact that other states in $\mathrm{S}$ satisfy the fixpoint at smaller iterates than $s$ does. So if one follows a chain of dependencies, the chain terminates. Therefore, $\mu$ means 'finite looping'. On the other hand, for a maximal fixpoint $\nu X . \phi$, there is no such decreasing chain: $s \models \nu Z$. $\phi$ iff $s \models \nu^{\beta} Z . \phi$ for every iterate $\beta$ iff $s=\phi\left(\nu^{\beta} Z . \phi\right)$ for every iterate $\beta$ iff $s=\phi(\nu Z . \phi)$, and so we may loop for ever.

We impose a further syntactic constraint on formulas. In the following we write $\sigma Z$. $\phi$ for $\mu Z . \phi$ or $\nu Z . \phi$ when we are indifferent to which fixpoint.

Definition 2. The formula $\gamma$ of $\mu M$ is guarded if for any subformula $\sigma Z$. $\phi$ of $\gamma$, every occurrence of $Z$ in $\phi$ is within the scope of a modal operator.

The following is standard; see [5, 9, 14].

Fact 2. Every formula of $\mu M$ is equivalent to a guarded formula.

\section{Proof Systems}

There has been a variety of proof systems for $\mu M$. Kozen presented an equational deductive system which is equivalent to the Henkin axiom system of Figure 1 that extends the standard modal logic $K$ [5]: here $\phi \rightarrow \psi$ means $\neg \phi \vee \psi$. There is an extra axiom for a least fixed point that its "unfolding" implies it; and Park's fixed point induction rule which says that $\mu$ is indeed the least pre-fixed point. The duals of this axiom and rule for greatest fixed points are; 
axioms and rules for minimal multi-modal logic $\mathrm{K}$

$$
\begin{gathered}
\phi(\mu X . \phi(X)) \rightarrow \mu X . \phi(X) \\
\frac{\phi(\psi) \rightarrow \psi}{\mu X . \phi(X) \rightarrow \psi}
\end{gathered}
$$

Figure 1: Kozen's axiomatisation of $\mu M$

$$
\begin{array}{cc}
\Gamma, P, \neg P & \Gamma, \mathrm{tt} \\
\frac{\Gamma, \phi \vee \psi}{\Gamma, \phi, \psi} & \frac{\Gamma, \phi \wedge \psi}{\Gamma, \phi} \Gamma, \psi \\
\frac{\Gamma,\langle a\rangle \Sigma,[a] \psi}{\Sigma, \psi} \\
\frac{\Gamma, \nu Z . \phi(Z)}{\Gamma, \phi(\nu Z . \phi(Z))} & \frac{\Gamma, \mu Z . \phi(Z)}{\Gamma, \phi(\mu Z . \phi(Z))}
\end{array}
$$

Figure 2: Goal directed proof rules

$\nu X . \phi(X) \rightarrow \phi(\mu X . \phi(X))$ and if $\psi \rightarrow \phi(\psi)$ then $\psi \rightarrow \nu X . \phi(X)$. Despite the naturalness of this axiomatisation, Kozen was unable to show that it was complete in [5. Instead, he proved it complete for a subset of $\mu M$, the aconjunctive fragment. Subsequently, he provided a complete infinitary deductive system for the whole of $\mu M$ by adding the following infinitary rule [6].

$$
\frac{\mu^{n} X . \phi(X) \rightarrow \psi \text { for all } n<\omega}{\mu X . \phi(X) \rightarrow \psi}
$$

Soundness of this rule depends on the finite model theorem which is that a formula is satisfiable if, and only if, it is satisfiable in a finite model; soundness of the decision procedure in [1] also relies on this theorem. It is possible to devise an infinite structure with state $s$ such that, for instance, $s \models \mu^{n} X$. [a]X $\rightarrow$ ff for all $n<\omega$ and $s=\mu X$. [a]X.

Later Walukiewicz established that indeed Kozen's axiomatisation in Figure 1 is complete for the whole language. The proof appeals to a normal form, disjunctive normal form, inspired by automata and semantic tableaux and also uses (a slightly weakened version of) aconjunctivity [14. First, it is shown that every formula is provably equivalent to a guarded formula (thereby strengthening Fact 2). For any unsatisfiable weakly aconjunctive or disjunctive normal form formula $\phi$ there is a proof of $\neg \phi$. Then the central argument proceeds by induction on formulas showing that every guarded formula provably implies a semantically equivalent disjunctive normal form formula. This unusual proof method for showing completeness can be contrasted with the more standard technique of building a model out of consistent sets of formulas (which has remained elusive for $\mu M$ ).

Given a valid formula such as $\nu Z . \mu X .[a] Z \vee\langle a\rangle X$ it is not so easy to provide a proof of it within Kozen's axiom system. This suggests that one may also seek natural deduction, sequent or tableau style proof systems. A goal directed proof system is presented in Figure2, A sequent of this proof system is a set of formulas understood disjunctively; we assume $\Gamma, \Sigma, \ldots$ indicate a set of formulas and $\Gamma, \phi, \psi$ is the set $\Gamma \cup\{\phi, \psi\}$; clearly, $\Gamma, P, \neg P$ and $\Gamma$, tt are then valid. The 


$$
\begin{gathered}
\frac{\nu Z . \mu X .[a] Z \vee\langle a\rangle X}{\mu X .[a](\nu Z . \mu X .[a] Z \vee\langle a\rangle X) \vee\langle a\rangle X} \\
\frac{[a](\nu Z . \mu X .[a] Z \vee\langle a\rangle X) \vee\langle a\rangle(\mu X .[a](\nu Z . \mu X .[a] Z \vee\langle a\rangle X) \vee\langle a\rangle X)}{[a](\nu Z . \mu X .[a] Z \vee\langle a\rangle X),\langle a\rangle(\mu X .[a](\nu Z . \mu X .[a] Z \vee\langle a\rangle X) \vee\langle a\rangle X)} \\
\frac{\nu Z . \mu X .[a] Z \vee\langle a\rangle X, \mu X .[a](\nu Z . \mu X .[a] Z \vee\langle a\rangle X) \vee\langle a\rangle X}{\frac{\mu X .[a](\nu Z . \mu X .[a] Z \vee\langle a\rangle X) \vee\langle a\rangle X}{: !}}
\end{gathered}
$$

Figure 3: A never ending proof tree

rules remove $\vee$ between formulas and branch at an $\wedge$. Some notation in the modal rule: $\langle a\rangle \Sigma$ is the set of formulas $\{\langle a\rangle \phi \mid \phi \in \Sigma\}$. In its application the set $\Sigma$ can be empty. Fixpoint formulas are unfolded. The idea is to build a proof for a starting guarded formula $\gamma$ in positive normal form. Such systems have been presented before. For instance, in 9 there is a dual system for showing that a formula is unsatisfiable. There are also systems, such as in 3, 4, 12, where the rules are inverted.

The main problem with the rules in Figure 2 is that they lead to infinite depth proof trees as in Figure 3. It is unclear when such a tree is in fact a proof; for instance, there are such trees for invalid formulas such as $\mu X .[a] X \vee\langle a\rangle X$. One solution is to replace infinite depth proofs with proofs of infinite width by adopting a variant of Kozen's infinitary rule. In [4, 12, the authors add an infinitary rule as follows (again whose soundness depends on the finite model theorem).

$$
\begin{gathered}
\frac{\Gamma, \nu Z . \phi(Z)}{\Gamma, \nu^{1} Z . \phi(Z) \ldots \Gamma, \nu^{n} Z . \phi(Z) \ldots} \\
\frac{\Gamma, \nu^{1} Z . \phi(Z)}{\Gamma, \phi(\mathrm{tt})} \quad \frac{\Gamma, \nu^{n+1} Z . \phi(Z)}{\Gamma, \phi\left(\nu^{n} Z . \phi(Z)\right)}
\end{gathered}
$$

Every branch in a successful proof tree thereby is finite and finishes at a sequent $\Gamma$, tt or $\Gamma, P, \neg P$. For instance, Figure 4 illustrates part of the proof tree for $\nu Z . \mu X$. $[a] Z \vee\langle a\rangle X$.

Alternatively, one can accept infinite depth proofs but find a finite way of generating or recognising them. Extra criteria for deciding when an infinite tree labelled with sets of formulas is indeed a proof are necessary. In particular, we need to guarantee (see comments after Fact 1) that in any infinite branch a greatest fixpoint formula is unfolded infinitely often. In [9] the authors add the extra mechanism of an infinite game that plays over an infinite tree. In 3 for linear time mu-calculus the extra mechanism is a nondeterministic parity automaton that runs over the tree.

What we shall do is to show that indeed there is a means for obtaining a finite proof using names. This mechanism was introduced in [8] as a tableau decision procedure for showing satisfiability of $\mu M$ formulas. Here we reformulate it as a proof system for showing when a formula is valid. 


$$
\begin{gathered}
Z^{i}=\nu^{i} Z . \mu X .[a] Z \vee\langle a\rangle X \text { for } i>0 \\
\frac{\nu Z . \mu X .[a] Z \vee\langle a\rangle X}{\frac{Z^{1}}{\mu X .[a] \operatorname{tt} \vee\langle a\rangle X}} \\
\frac{\frac{Z^{2}}{\mu X .[a] Z^{1} \vee\langle a\rangle X}}{\frac{[a] \mathrm{tt} \vee\langle a\rangle(\mu X .[a] \mathrm{tt} \vee\langle a\rangle X)}{[a] \mathrm{tt},\langle a\rangle(\mu X .[a] \mathrm{tt} \vee\langle a\rangle X)}} \frac{\frac{Z^{i+1}}{\mathrm{tt}}}{\frac{[a] Z^{1} \vee\langle a\rangle\left(\mu X .[a] Z^{1} \vee\langle a\rangle X\right)}{[a] Z^{1},\langle a\rangle(\mu X .[a] \mathrm{tt} \vee\langle a\rangle X)}} \frac{\frac{Z^{1}}{Z^{i}}}{\vdots}
\end{gathered}
$$

Figure 4: An infinitely wide proof tree

\section{Proof System with Names}

Our aim is now to build a proof system such that a formula has a finite proof tree if, and only if, it is valid. The proof system includes some auxiliary naming notation. Assume a starting guarded closed formula $\gamma$ in positive normal form.

Definition 3. If in $\gamma$ the subformula $\sigma_{1} Z . \psi$ is a proper subformula of $\sigma_{2} Y . \phi$ then $Y$ is more outermost than $Z$ (in $\gamma$ ). Variable $X$ is a variable in $\gamma$ if $\sigma X . \psi$ is a subformula of $\gamma$ and it is $a \nu$-variable if $\sigma$ is $\nu$.

We assume a fixed linear ordering $X_{1}, \ldots, X_{m}$ on all the distinct variables in $\gamma$ such that if $X_{i}$ is more outermost than $X_{j}$ then $i<j$. For instance, in a linear ordering for variables in $(\nu Z . \mu X .[a] Z \vee\langle a\rangle X) \wedge \mu Y$. [a] $Y$ the $\nu$-variable $Z$ must occur before $X$ whereas $Y$ can occur before or after it. For each $\nu$-variable $Z$ in $\gamma$ we assume a finite set $\left\{z_{1}, z_{2}, \ldots, z_{l}\right\}$ of names for $Z$ where $l$ is the length of $\gamma$.

The proof system has sequents of the form $w \vdash \Gamma$ where $w$ is a sequence of distinct names for $\nu$-variables and each element of $\Gamma$ has the form $\phi^{u}$ where $\phi$ is a formula (belonging to the closure of $\gamma$ ) and $u$ is a subsequence of $w$. The initial sequent is $\vdash \gamma$ with the empty sequence of names. If $v=n_{1} \ldots n_{k}$ is a sequence of names then $v(i), 1 \leq i \leq k$, is the element $n_{i}$.

Definition 4. Assume $X_{1}, \ldots, X_{m}$ is the fixed linear ordering of variables in $\gamma$ and $u, v, w$ are sequences of names of these variables where $u, v$ are subsequences of $w$.

1. We write $u<_{w} v$ if for some $j,(1) u(j)$ and $v(j)$ are names of the same variable and $u(j)$ occurs before $v(j)$ in $w$, and (2) $u(i)=v(i)$ for all $i<j$.

2. The sequence $u \uparrow X_{i}$ is the subsequence of $u$ that omits all names of the variables $X_{i+1}, \ldots, X_{n}$.

3. We write $u \sqsubset_{w} v$ if $u<_{w} v$ or there is a $\nu$-variable $X_{i}$ such that $v \uparrow X_{i}$ is a proper prefix of $u \uparrow X_{i}$.

The proof rules in Figure 5 are an elaboration of those in Figure 2, Again, sets of formulas are to be understood disjunctively; now formulas also carry sequences of names reflecting the history of unfoldings of greatest fixpoints. The $\vee$ and $\wedge$ rules are similar to before; the names 


$$
\begin{array}{cc}
w \vdash \Gamma, P^{u}, \neg P^{v} & w \vdash \Gamma, \mathrm{tt}^{u} \\
\frac{w \vdash \Gamma, \phi \vee \psi^{u}}{w \vdash \Gamma, \phi^{u}, \psi^{u}} & \frac{w \vdash \Gamma, \phi \wedge \psi^{u}}{w \vdash \Gamma, \phi^{u}} w \vdash \Gamma, \psi^{u} \\
\frac{w \vdash \Gamma,\langle a\rangle \Sigma,[a] \psi^{u}}{w^{\prime} \vdash \Sigma, \psi^{u}} & \frac{w \vdash \Gamma, \mu Z . \phi(Z)^{u}}{w^{\prime} \vdash \Gamma, \phi(\mu Z . \phi(Z))^{u \uparrow Z}} \\
\frac{w \vdash \Gamma, \nu Z . \phi(Z)^{u}}{w^{\prime} z_{i} \vdash \Gamma, \phi(\nu Z . \phi(Z))^{\left(u\lceil Z) z_{i}\right.}} z_{i} & \text { is the first name for } Z \text { not occurring in } w
\end{array}
$$

Figure 5: Goal directed proof rules with names

$$
\begin{gathered}
\text { Thin } \frac{w \vdash \Gamma, \phi^{u}, \phi^{v}}{w^{\prime} \vdash \Gamma, \phi^{u}} u \sqsubset_{w} v \\
\operatorname{Reset}_{z} \frac{w \vdash \Gamma, \phi_{1}^{u z z_{1} u_{1}}, \ldots, \phi_{k}^{u z z_{k} u_{k}}}{w^{\prime} \vdash \Gamma, \phi_{1}^{u z}, \ldots, \phi_{k}^{u z}} z \text { does not occur in } \Gamma
\end{gathered}
$$

Figure 6: Structural proof rules

index is passed to the components. In the modal rule we assume that $\langle a\rangle \Sigma$ is the set of formulas $\left\{\langle a\rangle \phi^{u} \mid \phi^{u} \in \Sigma\right\}$; in an application $\Sigma$ can be empty. Some further notation: $w^{\prime}$ in the conclusion of the modal rule (and in other rules) is the subsequence of names in $w$ that still occur in $\Sigma$ and $u$; names that occurred only in formulas in the premises $\Gamma$ are removed from $w$. Fixpoint formulas are unfolded; names in $u$ that belong to variables that are more innermost than $Z$ are removed from $u$ (and from $w$ if they do not occur in $\Gamma$ ). In the case of a greatest fixpoint a new name for $z$ is also added to the name sequence (both in $w^{\prime}$ and $u\lceil Z$ ). Importantly, there are also two key structural rules in Figure 6. If $\phi^{u}$ and $\phi^{v}$ both occur in a sequent $w \vdash \Sigma$ then either $u \sqsubset_{w} v$ or $v \sqsubset_{w} u$. In the case of the rule $\operatorname{Reset}_{z}$ the names $z, z_{1}, \ldots, z_{k}$ are names for the same variable $Z$ and $z_{i}$ could be the same as $z_{j}$. When applying the proof rules of Figures 5 and 6 we assume that the structural rules have priority over the logical rules.

Definition 5. A node $n$ of a tree labelled with the sequent $w \vdash \Gamma$ is a leaf if there is a node $m$ above it, its companion, labelled with the same sequent $w \vdash \Gamma$; this leaf is successful if between nodes $n$ and $m$ there is an application of the rule Reset $t_{z}$ for some $z$ such that for any node $n^{\prime}$ labelled with $w^{\prime} \vdash \Sigma$ between and including $n$ and $m$ the name $z$ occurs in $w^{\prime}$.

Definition 6. A proof tree for $\gamma$ is a tree where

1. the root is labelled $\vdash \gamma$,

2. any other node is labelled with a sequent that is the result of an application of a rule in Figure 5 or 6 to the sequent at its parent node,

3. each leaf is labelled with a sequent that is an instance of an axiom in Figure 5 or is successful according to the repeat condition.

A tree is not a proof if it has a leaf labelled with a sequent of the form

$$
w \vdash P_{1}^{u_{1}}, \ldots, P_{k}^{u_{k}}, \neg Q_{1}^{v_{1}}, \ldots, \neg Q_{l}^{v_{l}},\left\langle a_{1}\right\rangle \Sigma_{1}, \ldots,\left\langle a_{m}\right\rangle \Sigma_{m}
$$




$$
\begin{gathered}
Z=\nu Z . \mu X .[a] Z \vee\langle a\rangle X \quad X=\mu X .[a] Z \vee\langle a\rangle X \\
\frac{\frac{\vdash Z}{z_{1} \vdash X^{z_{1}}}}{\frac{z_{1} \vdash([a] Z \vee\langle a\rangle X)^{z_{1}}}{z_{1} \vdash[a] Z^{z_{1}},\langle a\rangle X^{z_{1}}}} \\
\frac{\frac{z_{1} \vdash Z^{z_{1}}, X^{z_{1}}}{z_{1} z_{2} \vdash X^{z_{1} z_{2}}, X^{z_{1}}}}{\frac{z_{1} z_{2} \vdash X^{z_{1} z_{2}}}{z_{1} \vdash X^{z_{1}}} \text { Rhin }} \text { Thet }_{z_{1}}
\end{gathered}
$$

Figure 7: A proof tree

where $Q_{j} \neq P_{i}$ for all $i, j$ or has a leaf $n$ that is a repeat because of its companion $m$ and for every application of a rule $\operatorname{Reset}_{z}$ between $m$ and $n$ there is a node $n^{\prime}$ between (and including) $n$ and $m$ labelled $w \vdash \Sigma$ such that $z$ does not occur in $w$. Given a formula $\gamma$ there are at most $2^{|\gamma|}$ different subsets of subformulas of $\gamma$ where $|\gamma|$ is the size of $\gamma$. The number of greatest fixpoints in $\gamma$ is also bounded by $|\gamma|$. The number of different possible sequents derivable from $\vdash \gamma$ is bounded by $2^{O\left(|\gamma|^{2}|\log (\gamma)|\right)}$, see [8, which is therefore also a bound on the depth of a tree. Moreover, the width of a tree is bounded by 2 . The only rule that allows choice is the modal rule; the number of choices is again bounded by $|\gamma|$. Therefore, the number of possible trees with root $\vdash \gamma$ is bounded in terms of $|\gamma|$.

Fact 3. For any closed guarded $\gamma$ there are only boundedly many trees for $\gamma$ and each such tree has boundedly many nodes (where the bounds are functions of $|\gamma|$ ).

In Figure 7 there is a proof tree for the valid formula $\nu Z . \mu X .[a] Z \vee\langle a\rangle X$ where we employ the abbreviations that $Z$ is this formula and $X$ is it's subformula $\mu X$. [a] $z \vee\langle a\rangle X$. It is a proof tree because of the repeat sequent $z_{1} \vdash X^{z_{1}}$ with an application of Repeat $z_{z_{1}}$ inbetween where $z_{1}$ is a name that occurs in each sequent throughout. The proof tree for a more complex valid formula $X \vee Z$ is illustrated in Figure 8 . We encourage the reader to check that indeed it is a proof tree.

At the cost of increasing the size of trees, we can add further conditions on when a node counts as a leaf in Definition 5. for instance, an extra requirement is that its sequent is the result of an application of the modal rule.

Theorem 4. For any closed guarded $\gamma, \models \gamma$ iff there is a proof tree for $\gamma$.

Proof. Assume $=\gamma$ but there is not a proof tree for $\gamma$. We show that we can build a countermodel to $\gamma$; a structure T and a state $s$ of T such that $s \not \models \gamma$. Given a sequent $w \vdash \Gamma$ it is valid if $\models \bigvee\left\{\phi \mid \exists u . \phi^{u} \in \Gamma\right\}$. The initial sequent $\vdash \gamma$ is valid. We now build a tree using the proof rules where each node is labelled with a valid sequent (or, as we shall see, a countermodel) and except for the root node is the result of an application of a proof rule. Assume we have built part of the tree and consider a current leaf labelled with a valid sequent; if it is not an 


$$
\begin{aligned}
& X=\nu X .\langle a\rangle X \wedge Y \quad Z=\nu Z \cdot[a] Z \vee W \\
& Y=\mu Y \cdot\langle a\rangle Y \vee P \quad W=\mu W \cdot[a] W \vee \neg P \\
& \vdash X, Z \\
& x_{1} \vdash(\langle a\rangle X \wedge Y)^{x_{1}}, Z \\
& \overline{x_{1} z_{1} \vdash(\langle a\rangle X \wedge Y)^{x_{1}},([a] Z \vee W)^{z_{1}}} \\
& x_{1} z_{1} \vdash(\langle a\rangle X \wedge Y)^{x_{1}},[a] Z^{z_{1}}, W^{z_{1}} \\
& \mathrm{~T} 1 \\
& x_{1} z_{1} \vdash Y^{x_{1}},[a] Z^{z_{1}}, W^{z_{1}} \\
& x_{1} z_{1} \vdash(\langle a\rangle Y \vee P)^{x_{1}},[a] Z^{z_{1}}, W^{z_{1}} \\
& x_{1} z_{1} \vdash\langle a\rangle Y^{x_{1}}, P^{x_{1}},[a] Z^{z_{1}}, W^{z_{1}} \\
& \overline{x_{1} z_{1} \vdash\langle a\rangle Y^{x_{1}}, P^{x_{1}},[a] Z^{z_{1}},([a] W \wedge \neg P)^{z_{1}}} \\
& x_{1} z_{1} \vdash\langle a\rangle Y^{x_{1}}, P^{x_{1}},[a] Z^{z_{1}},[a] W^{z_{1}} \quad x_{1} z_{1} \vdash\langle a\rangle Y^{x_{1}}, P^{x_{1}},[a] Z^{z_{1}}, \neg P^{z_{1}} \\
& x_{1} z_{1} \vdash Y^{x_{1}}, Z^{z_{1}} \\
& x_{1} z_{1} z_{2} \vdash Y^{x_{1}},([a] Z \vee W)^{z_{1} z_{2}} \\
& \frac{x_{1} z_{1} z_{2} \vdash Y^{x_{1}},[a] Z^{z_{1} z_{2}}, W^{z_{1} z_{2}}}{x_{1} z_{1} \vdash Y^{x_{1}},[a] Z^{z_{1}}, W^{z_{1}}} \operatorname{Reset}_{z_{1}}
\end{aligned}
$$

$\mathrm{T} 1$

$$
\frac{\overline{x_{1} z_{1} \vdash\langle a\rangle X^{x_{1}},[a] Z^{z_{1}}, W^{z_{1}}}}{x_{1} z_{1} \vdash\langle a\rangle X^{x_{1}},[a] Z^{z_{1}},([a] W \wedge \neg P)^{z_{1}}}
$$

$$
\begin{array}{cc}
\frac{x_{1} z_{1} \vdash\langle a\rangle X^{x_{1}},[a] Z^{z_{1}},[a] W^{z_{1}}}{x_{1} z_{1} \vdash X^{x_{1}}, Z^{z_{1}}} & \frac{x_{1} z_{1} \vdash\langle a\rangle X^{x_{1}},[a] Z^{z_{1}}, \neg P^{z_{1}}}{x_{1} z_{1} \vdash X^{x_{1}}, Z^{z_{1}}} \\
\frac{x_{1} z_{1} x_{2} \vdash(\langle a\rangle X \wedge Y)^{x_{1} x_{2}}, Z^{z_{1}}}{x_{1} z_{1} \vdash(\langle a\rangle X \wedge Y)^{x_{1}}, Z^{z_{1}}} \operatorname{Reset}_{x_{1}} & \frac{x_{1} z_{1} x_{2} \vdash(\langle a\rangle X \wedge Y)^{x_{1} x_{2}}, Z^{z_{1}}}{x_{1} z_{1} \vdash(\langle a\rangle X \wedge Y)^{x_{1}}, Z^{z_{1}}} \text { Reset }_{x_{1}} \\
\hline x_{1} z_{1} z_{2} \vdash(\langle a\rangle X \wedge Y)^{x_{1}},([a] Z \vee W)^{z_{1} z_{2}} & \operatorname{Reset}_{z_{1}} \\
x_{1} z_{1} \vdash(\langle a\rangle X \wedge Y)^{x_{1}},([a] Z \vee W)^{z_{1}} & \frac{x_{1} z_{1} z_{2} \vdash(\langle a\rangle X \wedge Y)^{x_{1}},([a] Z \vee W)^{z_{1} z_{2}}}{x_{1} z_{1} \vdash(\langle a\rangle X \wedge Y)^{x_{1}},([a] Z \vee W)^{z_{1}}} \operatorname{Reset}_{z_{1}}
\end{array}
$$

Figure 8: A proof tree 
axiom or a repeat then the tree can be extended with further valid sequents. This is clear if we can apply a structural rule of Figure 6 which has priority and it is also clear for $\wedge, \vee$ and the fixpoint rules of Figure 5 in all these cases if the premise sequent is valid then so are the conclusion sequents. We next come to the modal rule. We assume it is only applied if no other rule applies. Then a leaf of the current tree is labelled with a valid sequent of the form

$$
(*) \quad w \vdash P_{1}^{u_{1}}, \ldots, P_{k}^{u_{k}}, \neg Q_{1}^{v_{1}}, \ldots, \neg Q_{l}^{v_{l}},\left\langle a_{1}\right\rangle \Sigma_{1}, \ldots,\left\langle a_{m}\right\rangle \Sigma_{m},\left[b_{1}\right] \psi_{1}^{w_{1}}, \ldots,\left[b_{p}\right] \psi_{p}^{w_{p}}
$$

where each $\Sigma_{i}$ is nonempty, $a_{i} \neq a_{j}$ when $i \neq j$ and we assume it is not an axiom, so $P_{i} \neq Q_{j}$ for all $i, j$. A possible conclusion of an application of the modal rule has the form $w^{\prime} \vdash \Sigma_{i}, \psi_{j}^{w_{j}}$ when $a_{i}=b_{j}$ or $w^{\prime} \vdash \psi_{j}^{w_{j}}$ when $b_{j}$ is different from each $a_{i}$. With our tree we allow all such possible applications. For each such application if the sequent is not valid we let the node be a leaf and we associate a countermodel to it: that is, a structure $\mathbf{T}_{i j}$ and a state $s_{i j}$ such that $s_{i j} \not \models \bigvee\left\{\phi \mid \exists u . \phi^{u} \in \Sigma_{i}\right\} \vee \psi_{j}$ or a structure $\mathrm{T}_{j}$ and a state $s_{j}$ such that $s_{j} \not \models \psi_{j}$. If all possible applications of the rule are invalid, including the case when $p=0$ in $(*)$, then we obtain a contradiction by constructing a countermodel to the valid premise $(*)$ as follows. For T we take the disjoint union of each $\mathrm{T}_{i j}$ and of each $\mathrm{T}_{j}$ together with a new state $s$. For each $a_{i}$ such that $\neg \exists b_{j} . a_{i}=b_{j}$ assume there is not a transition of the form $s \stackrel{a_{i}}{\longrightarrow} s^{\prime}$. Otherwise, we let $s \stackrel{a_{i}}{\longrightarrow} s_{i j}$ of $\mathrm{T}_{i j}$ and $s \stackrel{b_{j}}{\longrightarrow} s_{j}$ of $\mathrm{T}_{j}$. Finally, we assume $s \notin \mathrm{V}_{\text {Prop }}\left(P_{i}\right)$ and $s \in \mathrm{V}_{\text {Prop }}\left(Q_{j}\right)$ for each $i, j$. Clearly, by construction, $s$ fails to satisfy each formula in $(*)$. Any node of the tree labelled with a sequent of the form $(*)$ is called a modal node. Therefore, there is at least one child node labelled with a valid sequent of a modal node. For each such child we continue to extend the tree. The tree building eventually stops when nodes are leaves either because they are children of a modal node labelled with an invalid sequent or nodes labelled with an axiom or a repeat node. In the last case we assume that we restrict repeat nodes to be children of modal nodes. All nodes of the tree except for some successors of modal nodes are labelled with valid sequents. However, by assumption there is not a proof tree for $\gamma$. We now prune the tree. Starting top down, at any node where $\wedge$ is applied we choose one of the successor nodes which fails to produce a proof tree; we discard the subtree of the other successor. The result is a finite tree where the only branching is at modal nodes. All leaves are either unsuccessful repeats or children of modal nodes labelled with invalid sequents (and with associated countermodels). From this tree we build a countermodel to $\gamma$. We identify as states any region of the tree starting at the root or at a child of a modal node labelled with a valid sequent down to, and including, the next modal node. In the case of a leaf that is a repeat we assume that there is a backward edge to its companion node above. If a state $s$ finishes at the modal node labelled with the sequent $(*)$ then for each $a_{i}$ such that $\neg \exists b_{j} . a_{i}=b_{j}$ assume there is not a transition of the form $s \stackrel{a_{i}}{\longrightarrow} s^{\prime}$. Otherwise, for each child that is labelled with an invalid sequent we let $s \stackrel{a_{i}}{\longrightarrow} s_{i j}$ of the countermodel $\mathrm{T}_{i j}$ or $s \stackrel{b_{j}}{\longrightarrow} s_{j}$ of the countermodel $\mathrm{T}_{j}$. For any child labelled with valid sequent $w^{\prime} \vdash \Sigma_{i}, \psi_{j}^{w_{j}}$ when $a_{i}=b_{j}$ whose associated state is $s^{\prime}$ we assume a transition $s \stackrel{a_{i}}{\longrightarrow} s^{\prime}$ or any child $w^{\prime} \vdash \psi_{j}^{w_{j}}$ whose associated state is $s^{\prime}$ we assume a transition $s \stackrel{b_{j}}{\longrightarrow} s^{\prime}$ : the associated state of a repeating leaf is that of its companion (the target of the backedge). Finally, we assume $s \notin \mathrm{V}_{\text {Prop }}\left(P_{i}\right)$ and $s \in \mathrm{V}_{\text {Prop }}\left(Q_{j}\right)$ for each $i, j$. We say that $\phi \in s$ if $\exists u . \phi^{u}$ belongs to some sequent in the region associated with $s$. The proof is completed by showing that if $\phi \in s$ then in the countermodel $s \not \models \phi$. Assume to the contrary that for some $s$ and $\phi, \phi \in s$ and $s \models \phi$. Clearly, then $\phi$ is not a literal, an atomic formula or the negation of an atomic formula. For a formula $\phi \in s$ we can follow it through the tree, passing between states and jumping from a leaf to its companion. If $\phi_{1} \wedge \phi_{2} \in s$ then by construction $\phi_{1} \in s$ or $\phi_{2} \in s$. If $\phi_{1} \vee \phi_{2} \in s$ then we can choose between $\phi_{1} \in s$ and $\phi_{2} \in s$. If $\langle a\rangle \phi \in s$ then we look at the 
modal node associated with $s$ : if there is not a $t$ such tht $s \stackrel{a}{\longrightarrow} t$ or only countermodels under $a$-transitions to $\phi$ then $s \not \forall\langle a\rangle \phi$. Otherwise, we can choose a $t$ such that $s \stackrel{a}{\longrightarrow} t$ and $\phi \in t$. Similarly, for $[b] \psi \in s$. If $\sigma Z . \phi \in s$ then $\phi(\sigma Z . \phi) \in s$. Therefore, if we follow $\phi \in s$ for $s \models \phi$ we obtain a finite or infinite sequence $\phi_{1} \in s_{1}, \phi_{2} \in s_{2}, \ldots, \phi_{n} \in s_{n}$ where $\phi_{1}=\phi, s_{1}=s$, there is a state transition when $\phi$ is a modal formula and for all $i, s_{i} \models \phi_{i}$. Clearly, the sequence cannot be finite ending at a literal or a modal formula. So, the sequence must be infinite. We show that the outermost fixpoint unfolded infinitely often is a least fixpoint which is a contradiction by Fact 1. For suppose it is a greatest fixpoint $\nu Z . \psi$ : then the sequence of formulas must have a subsequence of the form $\ldots, \nu Z . \psi^{u}, \psi(\nu Z . \psi)^{u^{\prime} z}, \ldots, \nu Z . \psi^{u^{\prime} z u_{1}}, \psi(\nu Z . \psi)^{u^{\prime} z z_{i}}, \ldots, \nu Z . \psi^{u^{\prime} z u_{2}}$ where $\operatorname{Reset}_{z}$ is applied and $z$ is defined throughout: that is, the sequence must pass through a successful repeat.

For soundness, assume that there is a proof tree for $\gamma$ but $\not \models \gamma$. Therefore, there is a proof tree with root labelled $\vdash \gamma$ all of whose leaves are either labelled with axioms or are successful repeats. A sequent $w \vdash \Gamma$ is not valid if $\not \models \bigvee\left\{\phi \mid \exists u . \phi^{u} \in \Gamma\right\}$. First, if the premise of an application of a rule is not valid then so is a conclusion. This is clear for the structural rules, for the $\vee$ rule and the fixpoint rules. In the case of $\wedge$, if the premise sequent is not valid then one of the successor sequents is not valid. In the case of the modal rule, if $\models \bigvee \Sigma \vee \Psi$ then by standard modal reasoning $\models \phi \vee\langle a\rangle \Sigma \vee[a] \psi$; so, if the premise sequent is not valid then neither is the conclusion in an application of the modal rule. Next we refine the argument by adding ordinal information. If $\not=\nu Z$. $\phi$ then using Fact 1 there is a least ordinal $\alpha$, a countermodel T and a state $s$ of $\mathrm{T}$ such that $s \not \models \nu^{\alpha} Z . \phi$. To do this, we slightly change the rules (as in fact used in Figures 7 and 8 by letting variables abbreviate the fixpoint subformulas of $\gamma$.

$$
\begin{gathered}
\frac{w \vdash \Gamma, \sigma Z . \phi(Z)^{u}}{w \vdash \Gamma, Z^{u}} \quad \frac{w \vdash \Gamma, Z^{u}}{w^{\prime} \vdash \Gamma, \phi(Z)^{u \uparrow Z} Z \text { is } \mu Z . \phi(Z)} \\
\frac{w \vdash \Gamma, Z^{u}}{w^{\prime} z_{i} \vdash \Gamma, \phi(Z)^{(u \uparrow Z) z_{i}}} z \text { is } \nu Z . \phi(Z) \text { and } z_{i} \text { is the first name for } Z \text { not occurring in } w
\end{gathered}
$$

So, formulas can contain variables. We associate ordinals with sequents by adding ordinals to names. Assume an invalid sequent $w \vdash \Gamma$ where $w=n_{1}, \ldots, n_{k}$. We extend $w$ to pairs $\left(n_{1}, \alpha_{1}\right), \ldots,\left(n_{k}, \alpha_{k}\right)$ where each $\alpha_{i}$ is an ordinal: if $\phi^{u} \in \Gamma$ and $u$ contains a name for $Z$ then the meaning of $Z$ in $\phi^{u}$ is $\nu^{\alpha_{i}} Z . \psi$ when $Z$ is $\nu Z . \psi$ and where $z_{i}$ is the last name for $z$ in $u$. We assume that the invalid sequent $w \models \Gamma$ remains invalid when greatest fixpoint subformulas are so interpreted. We maintain the following invariant in an ordinal sequence: if $w=\left(n_{1}, \alpha_{1}\right), \ldots,\left(n_{k}, \alpha_{k}\right), i<j$ and $n_{i}, n_{j}$ name the same variable $Z$ such that there is a formula $\phi^{u}$ such that $n_{i}, n_{j}$ both occur in $u$ then $\alpha_{i}>\alpha_{j}$. Moreover, we assume lexicographic ordering on ordinal sequences: if $w=\left(n_{1}, \alpha_{1}\right), \ldots,\left(n_{k}, \alpha_{k}\right)$ and $w^{\prime}=\left(n_{1}, \beta_{1}\right), \ldots,\left(n_{k}, \beta_{k}\right)$ then $w<w^{\prime}$ if for some $j, \alpha_{j}<\beta_{j}$ and for all $i<j, \alpha_{i}=\beta_{i}$. We are interested in a least ordinal interpretation which makes $w \vdash \Gamma$ invalid. Moreover, if a proof rule is applied to such a sequent then a conclusion is invalid under the ordinal interpretation; we minimise the ordinal sequence which makes the conclusion invalid with respect to the lexicographical ordering. This is clear for the $\vee$, Thin, $\wedge$, modal, $\sigma Z$ and least fixpoint variable $Z$ (where we lose ordinals for any inner $X$ such that $Z>X$ ) rules. In the case of the maximal fixpoint variable rule with premise $w \vdash \Gamma, Z^{u}$ if there is no name for $Z$ in $u$ then we know that there is a least $\alpha$ such that $w^{\prime}\left(z_{i}, \alpha\right) \vdash \Gamma, \phi(Z)^{(u \uparrow Z) z_{i}}$ is invalid where $z_{i}$ is a new name for $Z$. Otherwise, there is a name for $Z$ in $u$; suppose the last one is $z_{j}$ with ordinal $\alpha_{j}$. Since the fixpoint is unfolded we know that we can decrease the meaning of $Z^{u}$ by at least one; so for the invalid conclusion $w^{\prime}\left(z_{i}, \alpha\right) \vdash \Gamma, \phi(Z)^{(u \nmid Z) z_{i}} \alpha<\alpha_{j}$. Finally, we turn to the $\operatorname{Reset}_{z}$ rule with 
premise $w \vdash \Gamma, \phi_{1}^{u z z_{1} u_{1}}, \ldots, \phi_{k}^{u z z_{k} u_{k}}$ where $z$ does not occur in $\Gamma$ and $z, z_{1}, \ldots, z_{k}$ name the same variable. In $w$ we have $(z, \alpha)$ and later $\left(z_{1}, \alpha_{1}\right), \ldots,\left(z_{k}, \alpha_{k}\right)$ (in any order). By the invariant property it follows that $\alpha>\alpha_{i}$ for each $i$ and that $Z$ of $\phi_{j}$ has meaning $\nu^{\beta_{j}} Z$. $\phi$ for $\beta_{j} \leq \alpha_{j}$ (as $u_{j}$ may contain further names for $Z$ ). Let $\beta=\min \left\{\alpha_{1}, \ldots, \alpha_{k}\right\}$. Clearly, we can replace $(z, \alpha)$ in $w$ with $(z, \beta)$, remove all the names $z_{i} u_{i}$ such that $w^{\prime} \vdash \Gamma, \phi_{1}^{u z}, \ldots, \phi_{k}^{u z}$ is invalid. Given a proof tree for $\gamma$ we now follow a branch of invalid sequents down the tree minimising their ordinal interpretations of variables. Clearly, we cannot reach a leaf $w \vdash \Gamma, P^{u}, \neg P^{v}$ or $w \vdash \Gamma, \mathrm{tt}^{u}$ as these sequents are valid. Moreover, we cannot reach a successful repeat $w \vdash \Gamma$ with an application of Reset $_{z}$ in between when $z$ is in each sequent throughout. Consider the companion node with ordinal interpretation $w=\left(n_{1}, \alpha_{1}\right), \ldots,\left(n_{k}, \alpha_{k}\right)$ and the leaf node with interpreation $w^{\prime}=\left(n_{1}, \beta_{1}\right), \ldots,\left(n_{k}, \beta_{k}\right)$ : it follows that $w^{\prime}<w$ as at least the entry for $z$ was reduced by the Reset $_{z}$ rule which is a contradiction.

\section{Conclusion}

We have presented a sound and complete proof system for checking validity of modal mu-calculus formulas. However, it relies on auxiliary notation for names that keep track of unfoldings of greatest fixpoints.

We tried, but failed, to see if this method is able to underpin a different proof of completeness of Kozen's axiomatisation than Walukiewicz's proof by induction.

An alternative framework for deciding satisfiability and validity for $\mu M$ is automata-theoretic [11. Using two way automata there is also a decision procedure for satisfiability and validity of formulas when past modal operators are included [13. Neither a sound and complete axiom system nor a sound and complete tableau proof system have been developed for this extended fixpoint logic (which fails the finite model theorem).

\section{References}

[1] B. Banieqbal and H. Barringer, Temporal logic with fixed points. In Temporal Logic in Specification, LNCS 398 62-74 (1989).

[2] J. Bradfield and C. Stirling, Modal mu-calculi. In Handbook of Modal Logic ed. P. Blackburn, J. van Benthem and F. Wolter, 721-756, Elsevier (2007).

[3] C. Dax, M. Hofmann and M. Lange, A proof system for the linear time $\mu$-calculus. In Procs FSTTCS 2006 LNCS 4337 274-285 (2006).

[4] G. Jäger, M. Kretz and T. Studer, Canonical completeness of infinitary $\mu$. The Journaal of Logic and Algebraic Programming 76 270-292 (2008).

[5] D. Kozen, Results on the propositional $\mu$-calculus. Theor. Comput. Sci. 27 333-354 (1983).

[6] D. Kozen, A finite model theorem for the propositional $\mu$-calculus. Studia Logica $47233-241$ (1986).

[7] M. Lange and C. Stirling, Focus games for satisfiability and completeness of temporal logic. In Procs LICS 2001, 357-365 (2001).

[8] N. Jungteerapanich, A tableau system for the modal $\mu$-calculus. In Procs TABLEAUX 2009, LNAI 5607 220-234 (2009).

[9] D. Niwinski and I. Walukiewicz, Games for the $\mu$-calculus. Theor. Comput. Sci. 163 99-116 (1996).

[10] C. Stirling and D. Walker, Local model checking in the modal mu-calculus. Theor. Comput. Sci. 89 161-177 (1991). 
[11] R. Streett and E. Emerson, An automata theoretic decision procedure for the propositional mucalculus. Information and Computation 81 249-264 (1989).

[12] T. Studer, On the proof theory of the modal mu-calculus. Studia Logica 89 343-363 (2008).

[13] M. Vardi, Reasoning about the past with two-way automata. In Procs ICALP 98, LNCS 1443 628-641 (1998).

[14] I. Walukiewicz, Completeness of Kozen's axiomatisation of the propositional $\mu$-calculus. Information and Computation 157 142-182 (2000). 